\title{
CENTRAL EXTENSIONS OF TOPOLOGICAL CURRENT ALGEBRAS
}

\author{
PETER MAIER \\ Department of Mathematics, University of Technology Darmstadt \\ Schloßgartenstraße 7, D-64289 Darmstadt, Germany \\ E-mail:maier@mathematik.tu-darmstadt.de
}

\begin{abstract}
In this note we describe universal central extensions of certain topological current algebras, which in our context are algebras of type $A \otimes \mathfrak{g}$, where $\mathfrak{g}$ is a finite dimensional semisimple real Lie algebra and $A$ a certain commutative associative topological algebra.

Introduction. Although in the algebraic setting the problem to determine all central extensions of a given current algebra, i.e., a Lie algebra of type $A \otimes_{F} \mathfrak{g}$, where $F$ is any field and $A$ is some commutative associative unital $F$-algebra, is satisfactorily solved for $\operatorname{char}(F) \neq 2$ (see [6] for the case $\operatorname{char}(F)=0$ and [18] for the general case) not much is known if one deals with central extensions of topological Lie algebras. A topological result is contained in [3] but only for Lie algebras of type $C^{\infty}(M, \mathfrak{g}) \cong C^{\infty}(M) \otimes \mathfrak{g}$, where $M$ is a compact smooth manifold. In this note we construct universal central extensions for Lie algebras of type $\mathfrak{g} \otimes A$, where $\mathfrak{g}$ is a finite dimensional semisimple real Lie algebra and $A$ a commutative associative algebra in a suitable locally convex category. If $A$ equals the algebra $C^{\infty}(M)$ of smooth functions on a smooth finite dimensional manifold $M$ (without any compactness assumptions), we are able to give an explicite description of these universal extensions by using the $A$-module $\Omega^{1}(M)$ of smooth 1 -forms on $M$, thereby proving that this is the universal differential module for $A$ in the category of Fréchet $A$-modules. As a consequence of our results, we obtain a generalisation of a theorem due to Pressley and Segal. Furthermore, we obtain analogous results on universal central extensions of complete locally convex Lie algebras of type $C_{c}^{\infty}(M, \mathfrak{g}) \cong C_{c}^{\infty}(M) \otimes \mathfrak{g}$, where the subscript $c$ stands for compact support.

As an application we note that Theorem 16 and its corollaries form a good base in order to construct (universal) central extensions for current Lie groups or for certain topological current groups obtained from classical Lie groups by extending the ground
\end{abstract}

2000 Mathematics Subject Classification: 17B65, 17 B99.

Received 1 March 2001; revised 19 October 2001.

The paper is in final form and no version of it will be published elsewhere. 
field $\mathbb{R}$ or $\mathbb{C}$ to $A$. In [13] is shown that for a Lie group $H$ modeled on a sequentially complete locally convex (s.c.l.c.) space each 2-cocycle $\omega$ of the Lie algebra $\mathfrak{h}$ with values in some s.c.l.c. space $\mathfrak{z}$ can be integrated to a smooth local $\mathfrak{z}$-valued cocycle $f$ for $H$. In general one cannot expect these local cocycle $f$ to be smoothly extendable to the entire group $H$. Nevertheless, it makes sense to ask whether there exists a smooth global cocycle $\tilde{f}$ for $H$ which is a push-forward of $f$ with values in some quotient $\mathfrak{z} / \Gamma, \Gamma$ a discrete subgroup of $\mathfrak{z}$. The answer to this question depends on the behavior of the so-called period map which can be defined as follows: Let $\Omega$ be the left invariant 2 -form on $H$ with $\Omega_{1}=\omega$. Then the period map is defined by

$$
\operatorname{per}_{\omega}: \pi_{2}(H) \rightarrow \mathfrak{z}:[\gamma] \mapsto \int_{\gamma} \Omega
$$

In [13] it is shown that the existence of $\tilde{f}$ is equivalent to the discreteness of $\operatorname{im}\left(\operatorname{per}_{\omega}\right)$ in $\mathfrak{z}$. At least for Fréchet-Lie groups of type $C^{\infty}(M, G), M$ compact, there exist simple criteria in order to decide when this is the case (see [10] for more details).

As a notational convention we note that in this paper omitting the range in the notation of a function space means that the functions are considered to be real-valued.

Topological tensor, alternating, and symmetric products. Let $E$ and $F$ be locally convex topological vector spaces, and let $E \otimes F$ denote their algebraic tensor product (if nothing else is specified, tensor products are always taken over the reals). The projective topology on $E \otimes F$ is the finest topology for which the map

$$
(x, y) \mapsto x \otimes y: E \times F \rightarrow E \otimes F
$$

is continuous. We call the so-defined topological tensor product of two locally convex vector spaces the projective tensor product. It has the universal property stated in Theorem 1 below. In the sequel we give another description for the projective topology which shows that this topology, indeed, is a locally convex vector space topology. Moreover, the description below yields that for two metrizable spaces $E$ and $F$ the projective tensor product again is a metrizable space. Let $p$ be a seminorm on $E$ and let $q$ be a seminorm on $F$. We define the tensor product $p \otimes q$ of these seminorms by

$$
(p \otimes q)(z):=\inf \left\{\sum p\left(x_{k}\right) q\left(y_{k}\right) \mid \sum x_{k} \otimes y_{k}=z\right\} .
$$

It turns out that this again is a seminorm and moreover, if $\left(p_{i}\right)_{i \in I}$ and $\left(q_{j}\right)_{j \in J}$ are two families of seminorms which define the topologies on $E$ and $F$, respectively, then $\left(p_{i} \otimes q_{j}\right)_{(i, j) \in I \times J}$ is a family of seminorms which defines the projective topology on $E \otimes F$ (cf. [8], 15.1).

1. Theorem. Let $E, F$, and $G$ be locally convex, resp. metrizable, locally convex spaces, and let $\beta: E \times F \rightarrow G$ be a continuous bilinear map. Then there exists a unique continuous linear map $\bar{\beta}: E \otimes F \rightarrow G$ such that $\beta=\bar{\beta} \circ \otimes$.

In general the projective tensor product of complete spaces $E$ and $F$ fails to be complete. We write $E \widehat{\otimes} F$ for its completion. Because of the universal property of the completion of a (metrizable) locally convex vector space we immediately obtain the following consequence of Theorem 1. 
2. Corollary. Let $E, F$, and $G$ be complete, resp. completely metrizable, locally convex spaces, and let $\beta: E \times F \rightarrow G$ be a continuous bilinear map. Then there exists a unique continuous linear map $\bar{\beta}: E \widehat{\otimes} F \rightarrow G$ such that $\beta=\bar{\beta} \circ \otimes$.

In the sequel the letter $\mathbf{K}$ stands for the category of locally convex vector spaces with continuous linear maps as morphisms or for one of its full subcategories consisting of all complete, resp., metrizable, resp., completely metrizable (= Fréchet), spaces. Furthermore, given two spaces $E, F \in \mathbf{K}$ we write $E \otimes_{\mathbf{K}} F$ for tensor product having the universal property described in Theorem 1, resp., Corollary 2. Note that uniqueness of the map $\bar{\beta}$ arising in Theorem 1 and Corollary 2 implies that the algebraic tensor product $E \otimes F$ always is dense in $E \otimes_{\mathbf{K}} F$. Now let $E=F \in \mathbf{K}$, then the map

$$
\sigma: E \times E \rightarrow E \otimes_{\mathbf{K}} E:(x, y) \mapsto y \otimes x
$$

induces a continuous linear involution

$$
\bar{\sigma}: E \otimes_{\mathbf{K}} E \rightarrow E \otimes_{\mathbf{K}} E
$$

which yields a decomposition

$$
E \otimes_{\mathbf{K}} E=S_{\mathbf{K}}^{2}(E) \oplus \Lambda_{\mathbf{K}}(E),
$$

where $S_{\mathbf{K}}^{2}(E):=\operatorname{ker}(1-\bar{\sigma})$ and $\Lambda_{\mathbf{K}}(E):=\operatorname{ker}(1+\bar{\sigma})$. Putting

$$
f \vee g:=\frac{1}{2}(f \otimes g+g \otimes f) \quad \text { and } \quad f \wedge g:=\frac{1}{2}(f \otimes g-g \otimes f)
$$

we obtain as a consequence of Theorem 1 and Corollary 2 the following result:

3. Theorem. Let $E, F \in \mathbf{K}$. Then for any continuous symmetric, resp., skew-symmetric bilinear map $\beta: E \times E \rightarrow F$ there exists a uniquely determined continuous linear map

$$
\bar{\beta}: S_{\mathbf{K}}^{2}(E) \rightarrow F, \quad \text { resp. }, \quad \bar{\beta}: \Lambda_{\mathbf{K}}^{2}(E) \rightarrow F
$$

such that $\beta=\bar{\beta} \circ \vee$, resp., $\beta=\bar{\beta} \circ \wedge$.

Now, we consider a special situation which will be of interest for us later on. Let $M$ be a finite dimensional smooth manifold and $E$ a Fréchet space. We topologize the space $C^{\infty}(M, E)$ in the following way: For any two topological spaces $X$ and $Y$ we denote by $C(X, Y)_{c}$ the space $C(X, Y)$ endowed with the topology of compact convergence. We identify the tangent bundle TE of $E$ with $E \times E$, so that for any smooth map $f: M \rightarrow E$ we obtain a smooth map $d f: \mathrm{T} M \rightarrow E$ by letting $d f(v):=\operatorname{pr}_{2}(\mathrm{~T} f(v))$. Inductively, this yields maps $d^{n} f: T^{n} M \rightarrow E$ for any $n \in \mathbb{N}_{0}$ by putting $d^{0} f:=f\left(T^{0} M:=M\right)$ and $d^{n} f:=d\left(d^{n-1} f\right)$ for $n>0$. Using these maps, we get an injection

$$
C^{\infty}(M, E) \rightarrow \prod_{n \in \mathbb{N}_{0}} C\left(\mathrm{~T}^{n} M, E\right)_{c}: f \mapsto\left(d^{n} f\right)_{n \in \mathbb{N}_{0}} .
$$

We endow $C^{\infty}(M, E)$ with the topology induced by the product topology via this embedding. Since for each of the spaces $\mathrm{T}^{n} M, n \in \mathbb{N}_{0}$, the respective topology has a countable basis consisting of relatively compact neighborhoods, the topology of each space $C\left(\mathrm{~T}^{n} M, E\right)_{c}$ can be defined by a countable separating family of seminorms and therefore is locally convex and metrizable. As a subspace of a countable product of locally convex metrizable spaces the space $C^{\infty}(M, E)$ is locally convex and metrizable as well. 
In fact, it turns out that its topology even is complete (cf. the proof of Proposition III.1 in [14]) whence $C^{\infty}(M, E)$ is a Fréchet space. Now we restrict our attention to the special case where $E=\mathbb{R}$ and write $C^{\infty}(M)$ for $C^{\infty}(M, \mathbb{R})$. While the isomorphism $C^{\infty}(M) \widehat{\otimes} C^{\infty}(N) \cong C^{\infty}(M \times N)$ is well-known if $M$ and $N$ are open subsets of some $\mathbb{R}^{n}$ a proof for the general case in which $M$ and $N$ are smooth finite dimensional manifolds is not easy to find in the literature.

4. Theorem. Let $M$ and $N$ be smooth finite dimensional manifolds. Then the map

$$
C^{\infty}(M) \widehat{\otimes} C^{\infty}(N) \rightarrow C^{\infty}(M \times N): f \otimes g \mapsto((p, q) \mapsto f(p) g(q))
$$

is an isomorphism of Fréchet spaces.

Proof. We first recall some facts. Let $X, Y$, and $Z$ be Hausdorff topological spaces. For $f \in C(X \times Y, Z)$ and $x \in X$ we put $f_{x}:=(y \mapsto f(x, y)) \in C(Y, Z)$. It is well-known that the map

$$
\alpha: C(X \times Y, Z)_{c} \rightarrow C\left(X, C(Y, Z)_{c}\right)_{c}: f \mapsto\left(x \mapsto f_{x}\right)
$$

is a homeomorphism if $Y$ is locally compact, and since $\alpha$, obviously, is natural in $X$ and $Z$, we obtain that $C(Y, \cdot)_{c}$ is a right adjoint self functor of the category of Hausdorff topological spaces and thus preserves limits. For the remaining proof we note that, according to [14], Theorem III.4, the image of the map $\left.\alpha\right|_{C^{\infty}(M \times N)}$ is contained in $C^{\infty}\left(M, C^{\infty}(N)\right)$, and that for any Fréchet space $E$ the map

$$
C^{\infty}(M) \widehat{\otimes} E \rightarrow C^{\infty}(M, E): f \otimes x \mapsto f x
$$

is an isomorphism of Fréchet spaces (cf. [5], Chapter II, p. 81). Hence we are done, if we can show that the map

$$
\beta: C^{\infty}(M \times N) \rightarrow C^{\infty}\left(M, C^{\infty}(N)\right): f \mapsto \alpha(f)
$$

is an isomorphism of Fréchet spaces. Thanks to the Open Mapping Theorem for Fréchet spaces it suffices to show that $\beta$ is a continuous linear bijection. Clearly, $\beta$ is injective. For the prove of its surjectivity we have to show that for $g \in C^{\infty}\left(M, C^{\infty}(N)\right)$ we have $\alpha^{-1}(g) \in C^{\infty}(M \times N)$, i.e., that $\alpha^{-1}(g)$ is smooth at any point. Since the latter is a local property we can assume $M$ and $N$ to be open subsets of some $\mathbb{R}^{n}$; but in this case the assertion is already proved, see [17], Theorem 40.1. It remains to show continuity of $\beta$. By definition of the topology of $C^{\infty}\left(M, C^{\infty}(N)\right)$ the map $\beta$ is continuous exactly if for any $m \in \mathbb{N}_{0}$ the map

$$
\beta_{m}: C^{\infty}(M \times N) \rightarrow C\left(\mathrm{~T}^{m} M, C^{\infty}(N)\right)_{c}: f \mapsto d^{m} \beta(f)
$$

is continuous. Since $C^{\infty}(N)$ is embedded into the product $\prod_{n \in \mathbb{N}_{0}} C\left(\mathrm{~T}^{n} N\right)_{c}$, and since $C\left(\mathrm{~T}^{m} M, \cdot\right)$ preserves limits, the map $\beta_{m}$ is continuous exactly if for any $n \in \mathbb{N}_{0}$ the map

$$
\beta_{m n}: C^{\infty}(M \times N) \rightarrow C\left(\mathrm{~T}^{m} M, C\left(\mathrm{~T}^{n} N\right)_{c}\right)_{c}: f \mapsto d^{n} \circ \beta_{m}(f)
$$

is continuous. In view of the isomorphism $C\left(\mathrm{~T}^{m} M, C\left(\mathrm{~T}^{n} N\right)_{c}\right)_{c} \cong C\left(\mathrm{~T}^{m} M \times \mathrm{T}^{n} N\right)_{c}$ continuity of $\beta_{m n}$ is equivalent to continuity of the map

$$
C^{\infty}(M \times N) \rightarrow C\left(\mathrm{~T}^{m} M \times \mathrm{T}^{n} N\right)_{c}: f \mapsto d_{1}^{m} d_{2}^{n} f
$$

where $d_{1}$ and $d_{2}$ denote the respective "partial derivatives". But the latter is clearly fulfilled, since $f$ is smooth. 
Universal differential modules. In this section we point out that the concept of a universal differential module for a commutative associative algebra, which is well-known in the algebraic setting, not only makes sense, but even is a very useful tool, in a categorial framework. By an algebra object in the category $\mathbf{K}$, or simply a $\mathbf{K}$-algebra, we mean an object $A \in \mathbf{K}$ together with a morphism

$$
\mu: A \otimes_{\mathbf{K}} A \rightarrow A
$$

called multiplication. Suppose for the rest of this note that $A$ is a unital $\mathbf{K}$-algebra with commutative and associative multiplication. An $A$-module in the category $\mathbf{K}$ is an object $M$ together with a morphism

$$
\nu: A \otimes_{\mathbf{K}} M \rightarrow M
$$

that satisfies $\nu \circ\left(\operatorname{id}_{A} \otimes \nu\right)=\nu \circ\left(\mu \otimes \mathrm{id}_{M}\right)$ and $\nu(1 \otimes m)=m$ for each $m \in M$. A derivation from such an algebra $A$ into an $A$-module $M$ is defined to be a linear map $D: A \rightarrow M$ satisfying

$$
D(a b)=a D b+b D a
$$

for all $a, b \in A$. The embedding

$$
a \mapsto a \otimes 1: A \rightarrow A \otimes_{\mathbf{K}} A
$$

turns the $\mathbf{K}$-algebra $A \otimes_{\mathbf{K}} A$ into an $A$-module with respect to the multiplication map on $A \otimes_{\mathbf{K}} A$, and in view of this module structure the map $\bar{\mu}$ also is a morphism of $A$-modules. Consequently, its kernel $I$ is an $A$-submodule of $A \otimes_{\mathbf{K}} A$.

5. Lemma. Let $J:=I \cap(A \otimes A)$. Then we have $J=\operatorname{span}_{A}\{1 \otimes b-b \otimes 1 \mid b \in A\}$ and $I=\bar{J}$.

Proof. Obviously, we have $J^{\prime}:=\operatorname{span}_{A}\{1 \otimes b-b \otimes 1 \mid b \in A\} \subseteq J$. In order to show the reverse inclusion consider $c=\sum a_{k} \otimes b_{k} \in J$, that is, $\sum a_{k} b_{k}=0$. Then we have

$$
c=\sum a_{k} \otimes b_{k}-\left(\sum a_{k} b_{k}\right) \otimes 1=\sum a_{k}\left(1 \otimes b_{k}-b_{k} \otimes 1\right) \in J^{\prime},
$$

and the first claim follows. To prove the second claim we note that the map

$$
c \mapsto(c-\mu(c) \otimes 1, \mu(c)): A \otimes_{\mathbf{K}} A \rightarrow I \oplus A
$$

is an isomorphism of $\mathbf{K}$ - $A$-modules whose inverse is given by

$$
(b, a) \mapsto b+a \otimes 1: I \oplus A \rightarrow A \otimes_{\mathbf{K}} A .
$$

As a consequence of this, the map

$$
\lambda: A \otimes_{\mathbf{K}} A \rightarrow I: c \mapsto c-\mu(c) \otimes 1
$$

is a surjective morphism of $\mathbf{K}$ - $A$-modules satisfying $\lambda(A \otimes A)=J$, by what we have just shown. As $A \otimes A$ is dense in $A \otimes_{\mathbf{K}} A$, this implies the second claim.

While quotients of Fréchet spaces again are Fréchet spaces, quotients of arbitrary complete locally convex spaces need not be complete (cf. [9], §31.6). So it may happen that for a complete locally convex algebra $A$ the space $I / \overline{I^{2}}$ is not complete. For that reason we define $\Omega_{\mathbf{K}}^{1}(A)$ to be the space $I / \overline{I^{2}}$ except $\mathbf{K}$ is the category of complete locally 
convex spaces where we define $\Omega_{\mathbf{K}}^{1}(A)$ to be the completion of the space $I / \overline{I^{2}}$. Moreover, we define a continuous linear map $d_{A}: A \rightarrow \Omega_{\mathbf{K}}^{1}(A)$ by the prescription

$$
d_{A}(a):=[1 \otimes a-a \otimes 1]
$$

where $[c]$ denotes the class of an element $c \in I$ in $\Omega_{\mathbf{K}}^{1}(A)$. Since we have

$$
\begin{aligned}
d_{A}(a b)-a d_{A}(b)-b d_{A}(a) & =[1 \otimes a b-a b \otimes 1]-[a \otimes b-a b \otimes 1]-[b \otimes a-b a \otimes 1] \\
& =[1 \otimes a b-a \otimes b-b \otimes a+a b \otimes 1] \\
& =[(1 \otimes a-a \otimes 1)(1 \otimes b-b \otimes 1)] \\
& =0
\end{aligned}
$$

for all $a, b \in A$, we see that $d_{A}$ in fact is a derivation. We call the pair $\left(\Omega_{\mathbf{K}}^{1}(A), d_{A}\right)$ the $\mathbf{K}$-universal differential module of the algebra $A$. It has the following universal property:

6. Theorem. Let $E$ be a $\mathbf{K}-A$-module and let $D: A \rightarrow E$ be a continuous derivation. Then there exists a unique continuous A-linear map $\bar{D}: \Omega_{\mathbf{K}}^{1}(A) \rightarrow E$ such that $D=$ $\bar{D} \circ d_{A}$.

Proof. In order to prove the existence of the map $\bar{D}$, we consider the continuous bilinear map

$$
\Delta: A \times A \rightarrow F:(a, b) \mapsto a D b
$$

which induces a continuous linear map $\bar{\Delta}: A \otimes_{\mathbf{K}} A \rightarrow E$ satisfying

$$
\bar{\Delta}(a \otimes b)=a D b
$$

for all $a, b \in A$. As is easy to verify, this map fulfils the identity

$$
\bar{\Delta}\left(c c^{\prime}\right)=\mu(c) \bar{\Delta}\left(c^{\prime}\right)+\mu\left(c^{\prime}\right) \bar{\Delta}(c)
$$

for all $c, c^{\prime} \in A \otimes A$, and hence for all $c, c^{\prime} \in A \otimes_{\mathbf{K}} A$, because of the density of $A \otimes A$ in $A \otimes_{\mathrm{K}} A$. Equation (1) shows that $\bar{\Delta}$ vanishes on $I^{2}$ and thus on $\overline{I^{2}}$. Hence, the restriction $\left.\bar{\Delta}\right|_{I}$ factors to a map

$$
\bar{D}: \Omega_{\mathbf{K}}^{1}(A) \rightarrow E
$$

for which we have

$$
\left(\bar{D} \circ d_{A}\right)(a)=\bar{D}([1 \otimes a-a \otimes 1])=1 D a-a D 1=D a,
$$

as desired.

Uniqueness of the map $\bar{D}$ follows from the fact that, according to Lemma 5 , the image of $d_{A}$ generates a dense $A$-submodule of $\Omega_{\mathbf{K}}^{1}(A)$.

Now we consider a special situation. Let $M$ be a finite dimensional smooth manifold (which we always assume to be paracompact), $A:=C^{\infty}(M)$, resp., $B:=C^{\infty}(M \times M)$ the algebra of smooth functions on $M$, resp., $M \times M$, and let $A_{c}$ and $B_{c}$ the respective subalgebras consisting of compactly supported functions. Then $A$ and $B$ are Fréchet algebras whereas $A_{c}$ and $B_{c}$, viewed as subspaces, in general are just locally convex algebras. Denoting the category of Fréchet spaces by $\mathbf{F}$, we seek for a convenient description of the universal module $\Omega_{\mathbf{F}}^{1}(A)$. In the sequel we view the Fréchet algebra $B$ as a Fréchet $A$-module with respect to the embedding

$$
A \rightarrow B: f \mapsto((p, q) \mapsto f(p)) .
$$


Further, we consider the following morphisms of Fréchet $A$-modules:

$$
\delta^{*}: B \rightarrow A: F \mapsto F \circ \delta,
$$

where $\delta$ is the diagonal map $p \mapsto(p, p): M \rightarrow M \times M$, and

$$
\bar{\theta}: A \otimes_{\mathbf{F}} A \rightarrow B
$$

which is induced by the continuous bilinear map

$$
\theta: A \times A \rightarrow B:(f, g) \mapsto((p, q) \mapsto f(p) g(q))
$$

Both maps $\delta^{*}$ and $\bar{\theta}$ are also morphisms of the underlying Fréchet algebras and moreover, $\bar{\theta}$ is a homeomorphism according to Theorem 4. Denoting by $\mu: A \otimes_{\mathbf{F}} A \rightarrow A$ the multiplication map on $A$, we have

$$
\mu=\delta^{*} \circ \bar{\theta} .
$$

From this relation we immediately infer $K:=\operatorname{ker}\left(\delta^{*}\right)=\bar{\theta}(I)$ and therefore obtain the following isomorphism of Fréchet $A$-modules:

$$
\Omega_{\mathbf{F}}^{1}(A) \cong K / \overline{K^{2}} .
$$

In the sequel we think of $\Omega_{\mathbf{F}}^{1}(A)$ as $K / \overline{K^{2}}$ with respect to this isomorphism. Likewise, we identify $A \otimes_{\mathbf{F}} A$ with $B$ via $\bar{\theta}$. Now let T $M$ be the tangent bundle of $M$. Then the space $C^{\infty}(\mathrm{T} M)$ is a Fréchet $A$-module in which the space

$$
\Omega^{1}(M):=\left\{\alpha \in C^{\infty}(\mathrm{T} M)|(\forall p \in M) \alpha|_{\mathrm{T}_{p} M} \text { is linear }\right\}
$$

of smooth 1-forms on $M$ is a closed $A$-submodule and therefore is a Fréchet $A$-module as well. We denote the space of compactly supported 1-forms on $M$ by $\Omega_{c}^{1}(M)$. This space is a locally convex $A_{c}$-module as well as a locally convex $A$-module, and it is dense in $\Omega^{1}(M)$ since the identity element in $A$ is a limit of elements in $A_{c}$. We want to show that $\Omega^{1}(M)$ and $\Omega_{\mathbf{F}}^{1}(A)$ are isomorphic Fréchet $A$-modules, and in order to do this, we first collect some information on $\Omega_{c}^{1}(M)$.

We put $J_{c}:=\operatorname{ker}\left(\left.\delta^{*}\right|_{A_{c} \otimes A_{c}}\right)=K \cap\left(A_{c} \otimes A_{c}\right), K_{c}:=\operatorname{ker}\left(\left.\delta^{*}\right|_{B_{c}}\right)=K \cap B_{c}$ and consider the continuous linear map $\tau: B \rightarrow \Omega^{1}(M)$ defined by

$$
\tau(F)(x, X):=d F(x, x)(0, X) .
$$

As one easily checks, the map $\tau$ even is $A$-linear. In the subsequent proposition all closures are taken with respect to the topology induced by that of $B$.

7. Proposition. The kernel of $\left.\tau\right|_{J_{c}}$ equals $\overline{J_{c}^{2}} \cap J_{c}$, the kernel of $\left.\tau\right|_{K_{c}}$ equals $K_{c}^{2}$, and the kernel of $\left.\tau\right|_{K}$ equals $\overline{K^{2}}$.

Proof. We have

$$
\tau(F G)=\delta^{*}(F) \tau(G)+\delta^{*}(G) \tau(F)
$$

for all $F, G \in B$ (which is easily verified for $F, G \in A \otimes A$ and then follows by density). Hence, we have $\overline{J_{c}^{2}} \subseteq \operatorname{ker}\left(\left.\tau\right|_{J_{c}}\right), K_{c}^{2} \subseteq \operatorname{ker}\left(\left.\tau\right|_{K_{c}}\right)$ and $\overline{K^{2}} \subseteq \operatorname{ker}\left(\left.\tau\right|_{K}\right)$. It remains to show the reverse inclusions. Equation (2) also shows that $\operatorname{ker}\left(\left.\tau\right|_{J_{c}}\right)$ is an ideal in $A_{c} \otimes A_{c}$ and likewise that $\operatorname{ker}\left(\left.\tau\right|_{K_{c}}\right)$ and $\operatorname{ker}\left(\left.\tau\right|_{K}\right)$ are ideals in $B$. For the rest of this proof let $\left(U_{k}\right)_{k \in \mathbb{N}}$ be a locally finite open covering of $M$ consisting of relatively compact neighborhoods which are diffeomorphic to open convex 0-neighborhoods in $\mathbb{R}^{n}$ and let $\left(\varphi_{k}\right)_{k \in \mathbb{N}}$ be a 
partition of unity subordinate to this covering. Using this partition of unity on $M$ we obtain a partition of unity on $M \times M$ consisting of functions in $A_{c} \otimes A_{c}$ by choosing a bijection $\iota: \mathbb{N} \rightarrow \mathbb{N}^{2}$ and putting $\psi_{k}:=\varphi_{\iota_{1}(k)} \otimes \varphi_{\iota_{2}(k)}$. We first show that $\operatorname{ker}\left(\left.\tau\right|_{K_{c}}\right) \subseteq K_{c}^{2}$ and $\operatorname{ker}\left(\left.\tau\right|_{J_{c}}\right) \subseteq \overline{J_{c}^{2}}$. For that purpose we pick $F \in B_{c}$. Putting $F_{k}:=\psi_{k} F$ we have

$$
F=\sum_{k} F_{k}
$$

Since $\operatorname{supp}(F)$ is compact only finitely many of the supports of the functions $\psi_{k}$ intersect $\operatorname{supp}(F)$ so that we have $F=F_{1}+\ldots+F_{m}$ for some $m \in \mathbb{N}$. Since $\operatorname{ker}\left(\left.\tau\right|_{K_{c}}\right)$ is an ideal in $B_{c}$ it follows that

$$
F \in \operatorname{ker}\left(\left.\tau\right|_{K_{c}}\right) \quad \Longleftrightarrow \quad(\forall k \leq m) F_{k} \in \operatorname{ker}\left(\left.\tau\right|_{K_{c}}\right),
$$

and the fact $\operatorname{ker}\left(\left.\tau\right|_{J_{c}}\right)$ is an ideal in $A_{c} \otimes A_{c}$ implies

$$
F \in \operatorname{ker}\left(\left.\tau\right|_{J_{c}}\right) \quad \Longleftrightarrow \quad(\forall k \leq m) F_{k} \in \operatorname{ker}\left(\left.\tau\right|_{J_{c}}\right) .
$$

So it suffices to consider the case $M=\mathbb{R}^{n}$.

In order to prove the desired inclusion for this case, we define for each pair $(x, y) \in$ $\mathbb{R}^{n} \times \mathbb{R}^{n}$ functions $g_{(x, y)}, h_{(x, y)}: \mathbb{R} \rightarrow \mathbb{R}^{n} \times \mathbb{R}^{n}$ by

$$
g_{(x, y)}(t):=(t x+(1-t) y, y) \quad \text { and } \quad h_{(x, y)}(t):=(x, t x+(1-t) y) .
$$

Now let $F \in K_{c}$. Then we have

$$
\begin{aligned}
2 F(x, y) & =F(x, y)-F(y, y)+F(x, y)-F(x, x) \\
& =\int_{0}^{1}\left(F \circ g_{(x, y)}\right)^{\prime}(t) d t-\int_{0}^{1}\left(F \circ h_{(x, y)}\right)^{\prime}(t) d t
\end{aligned}
$$

and thus

$$
F(x, y)=\sum_{k=1}^{n}\left(x_{k}-y_{k}\right) F_{k}(x, y)
$$

where

$$
F_{k}(x, y):=\frac{1}{2} \int_{0}^{1}\left(\frac{\partial F}{\partial x_{k}}\left(g_{(x, y)}(t)\right)-\frac{\partial F}{\partial y_{k}}\left(h_{(x, y)}(t)\right)\right) d t
$$

and $x_{1}, \ldots, x_{n}, y_{1}, \ldots, y_{n}$ denote the coordinate functions on $M \times M$. Applying $\tau$ to equation (3) leads to the 1 -form

$$
\tau(F)=\left(x \mapsto-\sum_{k=1}^{n} F_{k}(x, x) d x_{k}\right)
$$

on $\mathbb{R}^{n}$ and we see that vanishing of $\tau$ on $F$ implies that any $F_{k}$ vanishes on the diagonal and so $F \in K$. We claim that each $F_{k}$ has compact support, but this easily follows from the definition, $\operatorname{since} \operatorname{supp}(F) \subseteq[-a, a]^{2 n}$ for $a \in \mathbb{R}$ implies

$$
\frac{\partial F}{\partial x_{k}} \circ g_{(x, y)}=\frac{\partial F}{\partial y_{k}} \circ h_{(x, y)}=0
$$

for $(x, y) \in \mathbb{R}^{2 n} \backslash[-a, a]^{2 n}$. Replacing the coordinate functions by functions $\bar{x}_{1}, \ldots, \bar{y}_{n}$ on $\mathbb{R}^{n}$ which coincide with the coordinate functions on $[-a, a]^{2 n}$ and vanish outside some compact neighborhood of $[-a, a]^{2 n}$ shows that $F \in K_{c}^{2}$. For $F \in \operatorname{ker}\left(\left.\tau\right|_{J_{c}}\right)$ one cannot expect the functions $F_{k}$ to be in $J_{c}$. In this case we proceed as follows: We consider the 
Fréchet subpaces $A_{a}:=\left\{g \in A \mid \operatorname{supp}(g) \subseteq[-a, a]^{n}\right\}$ and $B_{a}:=\{G \in B \mid \operatorname{supp}(G) \subseteq$ $\left.[-a, a]^{2 n}\right\}$ of $A$, resp., $B$. For these spaces the restriction of the map $\bar{\theta}: A \otimes_{\mathbf{F}} A \rightarrow B$ to the subspace $A_{a} \otimes_{\mathbf{F}} A_{a}$ is an isomorphism onto the space $B_{a}$ which allows us to identify both spaces. Lemma 5 now implies that each function $F_{k}$ is a limit of functions in $J_{c}$ with support in $[-a, a]^{2 n}$, so that we obtain

$$
F \in J_{c} \cdot \overline{J_{c}} \subseteq \overline{J_{c}^{2}} .
$$

Now let $F \in B$. We put

$$
F_{l}:=\left(\psi_{1}+\ldots+\psi_{l}\right) F
$$

and obtain a sequence $\left(F_{l}\right)_{l \in \mathbb{N}}$ in $B_{c}$ which converges to $F$ in $B$ (because of the local finiteness of the covering $\left.\left(U_{k}\right)_{k \in \mathbb{N}}\right)$. Now we have

$$
F \in \operatorname{ker}\left(\left.\tau\right|_{K}\right) \quad \Longleftrightarrow \quad(\forall l \in \mathbb{N}) F_{l} \in \operatorname{ker}\left(\left.\tau\right|_{K_{c}}\right),
$$

since $\operatorname{ker}\left(\left.\tau\right|_{K}\right)$ is an ideal in $B$ and $B_{c} K \subseteq K_{c}$. But since we already know that $\operatorname{ker}\left(\left.\tau\right|_{K_{c}}\right)=$ $K_{c}^{2}$ this implies $\operatorname{ker}\left(\left.\tau\right|_{K}\right) \subseteq \overline{K_{c}^{2}} \subseteq \overline{K^{2}}$, as desired.

8. Proposition. We have $\tau\left(B_{c}\right)=\tau\left(A_{c} \otimes A_{c}\right)=\Omega_{c}^{1}(M)$ and $\tau(B)=\Omega^{1}(M)$.

Proof. Clearly, we have $\tau\left(A_{c} \otimes A_{c}\right) \subseteq \tau\left(B_{c}\right)$ and so it suffices to show $\tau\left(A_{c} \otimes A_{c}\right)=$ $\Omega_{c}^{1}(M)$. So let $\alpha \in \Omega_{c}^{1}(M)$. First we consider the case that $M$ is diffeomorphic to an open convex subset $U$ of $\mathbb{R}^{n}$, where $n:=\operatorname{dim} M$. Then we have in local coordinates

$$
\alpha(p)=\sum_{k=1}^{n} f_{k}(p) d x_{k} .
$$

Choosing functions $\bar{x}_{1}, \ldots, \bar{x}_{n} \in C^{\infty}\left(\mathbb{R}^{n}\right)$ with compact support in $U$ which coincide with the coordinate functions on $\operatorname{supp}^{\prime}(\alpha)$, we obtain

$$
\alpha(p)=\sum_{k=1}^{n} f_{k}(p) d \bar{x}_{k}=\tau\left(\sum_{k=1}^{n} f_{k}(p) \otimes \bar{x}_{k}\right)
$$

and see that $\Omega_{c}^{1}(M)=\tau\left(A_{c} \otimes A_{c}\right)$ in this case. Now let $M$ be any finite dimensional manifold. By choosing a suitable partition of unity we get a decomposition $\alpha=\alpha_{1}+$ $\ldots+\alpha_{n}$ where each of the sets $\operatorname{supp}^{\prime}\left(\alpha_{k}\right)$ is contained in some neighborhood $U_{k}$ which is diffeomorphic to an open convex neighborhood in $\mathbb{R}^{n}$. Now $\tau\left(A_{c} \otimes A_{c}\right)=\Omega_{c}^{1}(M)$ follows by what we have just proved.

In order to prove $\tau(B)=\Omega^{1}(M)$ we choose a locally finite open covering $\left(U_{k}\right)_{k \in \mathbb{N}}$ of $M$ consisting of relatively compact neighborhoods which are diffeomorphic to open convex neighborhoods in $\mathbb{R}^{n}$. Furthermore, we choose a partition of unity $\left(\varphi_{k}\right)_{k \in \mathbb{N}}$ subordinate to this covering. Now let $\alpha \in \Omega^{1}(M)$ and put $\alpha_{k}:=\varphi_{k} \alpha \in \Omega_{c}^{1}(M)$ for each $k$. Then we have $\alpha=\sum_{k \in \mathbb{N}} \alpha_{k}$ in $\Omega^{1}(M)$. At the beginning of the proof we have seen that for any of these 1-forms $\alpha_{k}$ we find a function $F_{k} \in \Omega_{c}^{1}(M)$ with $\tau\left(F_{k}\right)=\alpha_{k}$ and $\operatorname{supp}\left(F_{k}\right) \subseteq U_{k} \times U_{k}$. We put $G_{k}:=F_{1}+\ldots+F_{k}$ for $k \in \mathbb{N}$ and note that the sequence $\left(G_{k}\right)_{k \in \mathbb{N}}$ converges to some $G$ in $B$ because of the local finiteness of $\left(U_{k}\right)_{k \in \mathbb{N}}$. Continuity of $\tau$ now yields $\tau(G)=\alpha$, and we are done.

9. Theorem. The map $\bar{d}: \Omega_{\mathbf{F}}^{1}(A) \rightarrow \Omega^{1}(M)$ induced by the differential $d: A \rightarrow$ $\Omega^{1}(M)$ is an isomorphism of Fréchet $A$-modules. 
Proof. Thanks to the Open Mapping Theorem for Fréchet spaces it suffices to show bijectivity of the map $\bar{d}$. With respect to the identification $\Omega_{\mathbf{F}}^{1}(A)=K / \overline{K^{2}}$ the injectivity of $\tau$ is equivalent to the equality $\operatorname{ker}\left(\left.\tau\right|_{K}\right)=\overline{K^{2}}$ and thus is an immediate consequence of Proposition 7. In order to show surjectivity, we have to show that $\tau(K)=\Omega^{1}(M)$. From Proposition 8 we know that $\tau(B)=\Omega^{1}(M)$. But this implies $\tau(K)=\Omega^{1}(M)$ since we have $\tau\left(F-\delta^{*}(F) \otimes 1\right)=\tau(F)$ and $F-\delta^{*}(F) \otimes 1 \in K$ for any $F \in B$.

10. REMARK. In fact, Theorem 9 seems to be well-known (although unproved in full strength, as far as the author knows) if $M$ is compact (cf. [2]).

Now let $\mathbf{C}$ be the category of complete locally convex spaces. As a strictly inductive limit of a cofinal sequence of Fréchet algebras of type $C_{X}^{\infty}(M):=\left\{f \in C^{\infty}(M) \mid \operatorname{supp}(f)\right.$ $\subseteq X\}, X \subseteq M$ compact, which are considered as subspaces of $C^{\infty}(M)$, the algebra $A_{c}=C_{c}^{\infty}(M)$ is an $L F$ algebra and hence, is complete (cf. [17]). Likewise, the $A_{c}$-module $\Omega_{c}^{1}(M)$ is a strictly inductive limit of spaces $\Omega_{X}^{1}(M):=\left\{\alpha \in \Omega^{1}(M) \mid \operatorname{supp}^{\prime}(\alpha) \subseteq X\right\}$, $X \subseteq M$ compact. In fact, the space $\Omega_{c}^{1}(M)$ even is a topological $A_{c}$-module with respect to this structure (cf. [4]). Since, in general, the algebra $A_{c}$ has no identity element, we write $A_{1}:=\mathbb{R} 1+A_{c}$. Now the question arises if the module $\Omega_{c}^{1}(M)$ is the universal differential module for the algebra $A_{1}$ in the category $\mathbf{C}$.

11. Theorem. The map $\bar{d}: \Omega_{\mathbf{C}}^{1}\left(A_{1}\right) \rightarrow \Omega_{c}^{1}(M)$ induced by the differential $d: A_{1} \rightarrow$ $\Omega_{c}^{1}(M)$ is an isomorphism of topological $A_{1}$-modules.

Proof. The assertion follows if we can show that the pair $\left(\Omega_{c}^{1}(M), d\right)$ has the property of a universal differential module for $A_{1}$ in the category $\mathbf{C}$. In order to obtain this, we first establish an algebraic description of $\Omega_{c}^{1}(M)$. For that purpose let $f, g \in A_{c}$ and let $\varepsilon \in A_{c}$ be a function that vanishes on a compact neighbourhood of $\operatorname{supp}(f) \cup \operatorname{supp}(g)$ and that equals 1 on $\operatorname{supp}(f) \cup \operatorname{supp}(g)$. Then we have $f \otimes g-f g \otimes \varepsilon \in J_{c}$ and $\tau(f \otimes g-f g \otimes \varepsilon)=$ $\tau(f \otimes g)$. Both relations together with Proposition 8 imply $\tau\left(J_{c}\right)=\tau\left(A_{c} \otimes A_{c}\right)=\Omega_{c}^{1}(M)$. Together with Proposition 7 this yields the following (algebraic) isomorphism of $A_{1}$ modules:

$$
\frac{J_{c}}{J_{c} \cap \overline{J_{c}^{2}}} \cong \Omega_{c}^{1}(M) .
$$

Now let $E$ be any complete locally convex topological $A_{1}$-module and let $D: A_{1} \rightarrow E$ be a continuous derivation. Using the facts we have just proved, we see as in the proof of Theorem 6 that the map

$$
\Delta: A_{1} \otimes A_{1} \rightarrow E: f \otimes g \rightarrow f D g
$$

induces an $A_{1}$-linear map

$$
\bar{D}: \frac{J_{c}}{J_{c} \cap \overline{J_{c}^{2}}} \cong \Omega_{c}^{1}(M) \rightarrow E, \quad \bar{D}(f d g)=\bar{D}(\tau(f \otimes g))=f D g .
$$

Obviously, we have $D=\bar{D} \circ d$, and uniqueness of $\bar{D}$ follows from the fact that $d A_{1}=d A_{c}$ generates $\Omega_{c}^{1}(M)$ as an $A_{1}$-module. It remains to show continuity of $\bar{D}$.

As the topology on $\Omega_{c}^{1}(M)$ is the direct limit topology with respect to the subspaces $\Omega_{X}^{1}(M), X \subseteq M$ compact, it suffices to verify that the restrictions $\left.\bar{D}\right|_{\Omega_{X}^{1}(M)}$ are continuous. For that purpose we choose on $M$ a partition of unity $\left(\varphi_{k}\right)_{k \in \mathbb{N}}$ as in the proof 
of Proposition 8. The set $\mathbb{N}_{X}:=\left\{k \in \mathbb{N} \mid \operatorname{supp}\left(\varphi_{j}\right) \cap X \neq \emptyset\right\}$ is finite, and for each $\alpha \in \Omega_{X}^{1}(M)$ we have

$$
\alpha=\sum_{k \in \mathbb{N}_{X}} \varphi_{k} \alpha=\sum_{k \in \mathbb{N}_{X}} \sum_{l} \alpha_{l}^{k} d \bar{x}_{l}^{k},
$$

where the functions $\bar{x}_{l}^{k}$ are suitably chosen as in the proof of Proposition 7 . Since the sum is finite, we obtain

$$
\bar{D}(\alpha)=\sum_{k \in \mathbb{N}_{X}} \bar{D}\left(\varphi_{k} \alpha\right)=\sum_{k \in \mathbb{N}_{X}} \sum_{l} \alpha_{l}^{k} D\left(\bar{x}_{l}^{k}\right) .
$$

Independence of the functions $\bar{x}_{l}^{k}$ of $\alpha$ and the fact that multiplication with $\varphi_{k}$ is a continuous endomorphism of $\Omega_{c}^{1}(M)$ implies that the maps

$$
\Omega_{c}^{1}(M) \rightarrow A: \alpha \mapsto \alpha_{l}^{k}
$$

are continuous. Continuity of the module structure on $E$ now yields continuity of $\bar{D}$.

The continuous case. An opposite to the smooth situation we are concerned with in the preceding discussion is the continuous case. Given a compact topological space $X$, one may ask for a universal differential module for the Banach algebra $A:=C(X)$ in the category of Banach $A$-modules. Indeed, such an object exists, and can be obtained by our general construction described in the previous section. Surprisingly, this construction always leads to the trivial module, as we shall see in the sequel. In order to show this, we introduce the notion of an amenable Banach algebra. Let $A$ be a Banach algebra. For any Banach $A$-bimodule $M$ the dual Banach space $M^{\prime}$ also carries the structure of an $A$-bimodule via

$$
(a f)(x):=f(x a) \quad \text { and } \quad(f a)(x):=f(a x)
$$

for $a \in A, x \in M$, and $f \in M^{\prime}$. We call $A$ amenable if for any $A$-bimodule $M$ and any continuous derivation $\delta: A \rightarrow M^{\prime}$ there exists $f \in M^{\prime}$ such that

$$
\delta(a)=a f-f a
$$

for all $a \in A$. Such derivations of $A$ are called inner $M^{\prime}$-derivations. In [1] the following is shown:

12. THEOREM. If $X$ is a compact topological space, then $C(X)$ is an amenable Banach algebra and furthermore, for any $C(X)$-bimodule $M$ each $M$-derivation of $C(X)$ is inner.

Proof. See [1], Theorem VI.12, and Proposition VI.14.

As an immediate consequence of Theorem 12 we now obtain the following result:

13. Corollary. If $X$ is a compact topological space, then the universal differential module for the Banach algebra $C(X)$ in the category of all Banach spaces is trivial.

Proof. Let $A:=C(X)$ and let $\Omega_{\mathbf{B}}^{1}(A)$ denote the universal differential module for $A$ in category of Banach spaces. We define on $\Omega_{\mathbf{B}}^{1}(A)$ an $A$-bimodule structure by $\omega a:=a \omega$. Theorem 12 now implies that each $\Omega_{\mathbf{B}}^{1}(A)$-derivation is inner, but with respect to the above defined bimodule structure on $\Omega_{\mathbf{B}}^{1}(A)$, any such inner derivation obviously is trivial, which implies that $\Omega_{\mathbf{B}}^{1}(A)$ itself is trivial, because it is generated by the range of a derivation. 
Finally we note that the situation even changes if we consider a compact $C^{1}$-manifold $M$, since in this case the differential $d: M \rightarrow \Omega_{0}^{1}(M)$ (where $\Omega_{0}^{1}(M)$ denotes the Banach space of continuous 1-forms on $M$ ) is a non-trivial derivation, and thus the respective universal differential module has to be non-trivial.

Central extensions of topological current algebras. Throughout this section the letter $A$ denotes a commutative associative unital $\mathbf{K}$-algebra. We mainly investigate central extensions of $\mathbf{K}$-current algebras, which are Lie algebras of type $A \otimes \mathfrak{g}$, where $\mathfrak{g}$ is some finite dimensional semisimple Lie algebra. Nevertheless, we start a bit more general. We are only interested in extensions that are described by continuous Lie algebra cocycles. Given a $\mathbf{K}$-Lie algebra $\mathfrak{g}$, an abelian $\mathbf{K}$-Lie algebra $\mathfrak{z}$, and a continuous 2-cocycle $\omega: \mathfrak{g} \times \mathfrak{g} \rightarrow \mathfrak{z}$, we write $\mathfrak{g} \oplus_{\omega} \mathfrak{z}$ for the Lie algebra $\mathfrak{g} \times \mathfrak{z}$ with the bracket

$$
[(x, a),(y, b)]:=([x, y], \omega(x, y)) .
$$

These extensions are exactly those which are given by an exact sequence

$$
0 \longrightarrow \mathfrak{z} \stackrel{\iota}{\longrightarrow} \mathfrak{h} \stackrel{\pi}{\longrightarrow} \mathfrak{g} \longrightarrow 0
$$

of K-Lie algebras in which the map $\pi$ admits a continuous linear section. Such an extension is called weakly universal if for any other central extension $\mathfrak{g} \oplus_{\eta} \mathfrak{y}$ there exists a morphism of K-Lie algebras $\varphi: \mathfrak{z} \rightarrow \mathfrak{y}$ such that $\eta=\varphi \circ \omega$, it is called universal if the morphism $\varphi$ is unique. In any of these cases $\omega$ is called a universal cocycle. We note that a weakly universal extension $\mathfrak{g} \oplus_{\omega} \mathfrak{z}$ is universal if $\mathfrak{g}$ is perfect (cf. [11], 1.9, Proposition 1).

We start by stating a general result on perfect Fréchet-Lie algebras. So let $\mathfrak{g}$ be a perfect Fréchet-Lie algebra. The Lie bracket induces a continuous linear map

$$
\beta: \Lambda_{\mathbf{F}}^{2}(\mathfrak{g}) \rightarrow \mathfrak{g}
$$

which, because of the Jacobian identity, factors to a map

$$
\bar{\beta}: \Lambda_{\mathbf{F}}^{2}(\mathfrak{g}) / B_{2}^{\mathbf{F}}(\mathfrak{g}) \rightarrow \mathfrak{g},
$$

where $B_{2}^{\mathbf{F}}(\mathfrak{g})$ denotes the closure of the span of all elements of the form

$$
x \wedge[y, z]+y \wedge[z, x]+z \wedge[x, y]
$$

in $\Lambda_{\mathbf{F}}^{2}(\mathfrak{g})$. Writing $\tilde{\mathfrak{g}}:=\Lambda_{\mathbf{F}}^{2}(\mathfrak{g}) / B_{2}^{\mathbf{F}}(\mathfrak{g})$ and $[x]:=x+B_{2}^{\mathbf{F}}(\mathfrak{g})$ for $x \in \Lambda_{\mathbf{F}}^{2}(\mathfrak{g})$ the prescription

$$
[[x],[y]]:=[x] \wedge[y]
$$

defines a continuous Lie bracket on the space $\widetilde{\mathfrak{g}}$. Denoting the kernel of the map $\bar{\beta}$ by $Z_{2}^{\mathbf{F}}(\mathfrak{g})$ and writing $H_{2}^{\mathbf{F}}(\mathfrak{g}):=Z_{2}^{\mathbf{F}}(\mathfrak{g}) / B_{2}^{\mathbf{F}}(\mathfrak{g})$, we have the following result which is a special case of [15], Corollary II.12, resp., Theorem III.8:

14. THEOREM. The perfect Fréchet-Lie algebra $\mathfrak{g}$ possesses a weakly universal central extension if there exists a continuous projection $p: \widetilde{\mathfrak{g}} \rightarrow H_{2}^{\mathbf{F}}(\mathfrak{g})$. In this case a weakly universal extension is given by the Fréchet-Lie algebra $\widetilde{\mathfrak{g}}$ with universal cocycle

$$
\omega: \mathfrak{g} \times \mathfrak{g} \rightarrow H_{2}^{\mathbf{F}}(\mathfrak{g}):(x, y) \mapsto p([x \wedge y]) .
$$

If, moreover, $\mathfrak{g}$ is a perfect Banach-Lie algebra, then the existence of a weakly universal central extension is equivalent to the existence of a continuous projection $p: \widetilde{\mathfrak{g}} \rightarrow H_{2}^{\mathbf{F}}(\mathfrak{g})$. 
From now on let $\mathfrak{g}$ be finite dimensional and semisimple. We put $\mathfrak{g}_{A}:=A \otimes \mathfrak{g}$ and define a Lie bracket on $\mathfrak{g}_{A}$ by

$$
[a \otimes x, b \otimes y]:=a b \otimes[x, y]
$$

With respect to this bracket $\mathfrak{g}_{A}$ becomes a perfect $\mathbf{K}$-Lie algebra. If $A$ is a Fréchet algebra, then Theorem 14 yields both a criterion for the existence of a weakly universal central extension of $\mathfrak{g}_{A}$ as well as a construction method for it. In the sequel we will see that such an extension not even always exists but also can be described in a more explicit way.

We consider the action of $\mathfrak{g}$ on $S^{2}(\mathfrak{g})$ given by

$$
x(y \vee z):=[x, y] \vee z+y \vee[x, z],
$$

put $V(\mathfrak{g}):=S^{2}(\mathfrak{g}) / \mathfrak{g} S^{2}(\mathfrak{g})$, and define a symmetric bilinear map $\kappa: \mathfrak{g} \times \mathfrak{g} \rightarrow V(\mathfrak{g})$ by

$$
\kappa(x, y):=[x \vee y],
$$

where $[z]$ denotes the class of an element $z \in S^{2}(\mathfrak{g})$ in $V(\mathfrak{g})$. Since we have

$$
\kappa([x, y], z)+\kappa(y,[x, z])=[[x, y] \vee z+y \vee[x, z]]=[x(y \vee z)]=0 .
$$

for all $x, y, z \in \mathfrak{g}$, this map is invariant. Furthermore, it has the following universal property:

15. Lemma. Let $E$ be a Fréchet space and $\beta: \mathfrak{g} \times \mathfrak{g} \rightarrow E$ a continuous invariant symmetric bilinear map. Then there exists a unique (continuous) linear map $\bar{\beta}: V(\mathfrak{g}) \rightarrow E$ such that $\beta=\bar{\beta} \circ \kappa$.

Proof. Uniqueness of $\bar{\beta}$ is clear. For the proof of the existence we note that, because of the symmetry of $\beta$, the universal property of $S^{2}(\mathfrak{g})$ yields a linear map $\widetilde{\beta}: S^{2}(\mathfrak{g}) \rightarrow E$ with $\widetilde{\beta}(x \vee y)=\beta(x, y)$. The invariance of $\beta$ then implies that $\mathfrak{g} S^{2}(\mathfrak{g})$ is contained in the kernel of $\widetilde{\beta}$, whence $\widetilde{\beta}$ factors to the desired map $\bar{\beta}: V(\mathfrak{g}) \rightarrow E$.

Now we put $\mathfrak{z}_{A}:=V(\mathfrak{g}) \otimes\left(\Omega_{\mathbf{K}}^{1}(A) / \overline{d_{A} A}\right)$ and define a map $\omega_{A}: \mathfrak{g}_{A} \times \mathfrak{g}_{A} \rightarrow \mathfrak{z}_{A}$ by

$$
\omega_{A}(f \otimes x, g \otimes y):=\kappa(x, y) \otimes\left[f d_{A}(g)\right],
$$

where $[\alpha]$ denotes the class of $\alpha \in \Omega_{\mathbf{K}}^{1}(A)$ in $\Omega_{\mathbf{K}}^{1}(A) / \overline{d_{A} A}$. Taking the invariance of $\kappa$ into account, this map is easily verified to be a continuous 2-cocycle on $\mathfrak{g}_{A}$ and hence defines a central extension of $\mathfrak{g}_{A}$. For this central extension we have the following result:

16. ThEOREM. If the Lie algebra $\mathfrak{g}$ is semisimple, then the Lie algebra $\widetilde{\mathfrak{g}}_{A}:=\mathfrak{g}_{A} \oplus_{\omega_{A}} \mathfrak{z} A$ is a universal central extension of $\mathfrak{g}_{A}$ in the category of $\mathbf{K}$-Lie algebras.

Proof. We note that the central extension $\widetilde{\mathfrak{g}}_{A}$ is automatically universal if it is weakly universal, since $\mathfrak{g}_{A}$ is perfect. So it remains to show that $\omega_{A}$ is a universal cocycle. Before doing this we fix some notation. For real vector spaces $E$ and $F$ and $k \in \mathbb{N}$ we denote by $\operatorname{Lin}^{k}(E, F)$ the space of $k$-linear $F$-valued mappings on $E$ and by $\operatorname{Alt}^{k}(E, F)$ its subspace of alternating mappings. If, moreover, $E$ is a $\mathfrak{g}$-module we write

$$
E_{\mathrm{fix}}:=\{v \in E \mid \mathfrak{g} v=\{0\}\} \quad \text { and } \quad E_{\text {eff }}:=\operatorname{span}\{x v \mid x \in \mathfrak{g}, v \in E\} .
$$

Identifying $\mathfrak{g}$ with $1 \otimes \mathfrak{g} \subseteq \mathfrak{g}_{A}$, an action of $\mathfrak{g}_{A}$ on $\operatorname{Lin}^{2}\left(\mathfrak{g}_{A}, \mathfrak{z}\right)$ is given by

$$
(x \beta)(y, z)=-\beta([x, y], z)-\beta(y,[x, z])
$$


for $\beta \in \operatorname{Lin}^{2}\left(\mathfrak{g}_{A}, \mathfrak{z}\right), x \in \mathfrak{g}$, and $y, z \in \mathfrak{g}_{A}$. Making use of the natural isomorphisms

$$
\begin{aligned}
\operatorname{Lin}^{2}\left(\mathfrak{g}_{A}, \mathfrak{z}\right) & \cong \operatorname{Lin}\left(\mathfrak{g}_{A} \otimes_{\mathbf{K}} \mathfrak{g}_{A}, \mathfrak{z}\right) \cong \operatorname{Lin}\left((\mathfrak{g} \otimes \mathfrak{g}) \otimes\left(A \otimes_{\mathbf{K}} A\right), \mathfrak{z}\right) \\
& \cong(\mathfrak{g} \otimes \mathfrak{g})^{*} \otimes \operatorname{Lin}\left(A \otimes_{\mathbf{K}} A, \mathfrak{z}\right)
\end{aligned}
$$

this action transforms to an action of $\mathfrak{g}$ on the space $(\mathfrak{g} \otimes \mathfrak{g})^{*} \otimes \operatorname{Lin}\left(A \otimes_{\mathbf{K}} A, \mathfrak{z}\right)$ which is trivial on the second factor and thus semisimple. Hence, $\operatorname{Lin}^{2}\left(\mathfrak{g}_{A}, \mathfrak{z}\right)$ and each of its submodules is a semisimple $\mathfrak{g}$-module. Consequently, for the submodule $Z_{\mathbf{K}}^{2}\left(\mathfrak{g}_{A}, \mathfrak{z}\right)$ of continuous $\mathfrak{z}$-valued 2-cocycles we have

$$
Z_{\mathbf{K}}^{2}\left(\mathfrak{g}_{A}, \mathfrak{z}\right)=Z_{\mathbf{K}}^{2}\left(\mathfrak{g}_{A}, \mathfrak{z}\right)_{\text {fix }} \oplus Z_{\mathbf{K}}^{2}\left(\mathfrak{g}_{A}, \mathfrak{z}\right)_{\text {eff }} .
$$

Furthermore, we have for any $x \in \mathfrak{g}_{A}$ and any $k \in \mathbb{N}$ the insertion map

$$
i(x): \operatorname{Alt}^{k}\left(\mathfrak{g}_{A}, \mathfrak{z}\right) \rightarrow \operatorname{Alt}^{k-1}\left(\mathfrak{g}_{A}, \mathfrak{z}\right): \alpha \mapsto \alpha(x, \cdot) .
$$

Taking $\omega \in Z_{\mathbf{K}}^{2}\left(\mathfrak{g}_{A}, \mathfrak{z}\right)$ the Cartan formula yields

$$
x \omega=d(i(x) \omega)+i(x)(d \omega)=d(i(x) \omega) \in B_{\mathbf{K}}^{2}\left(\mathfrak{g}_{A}, \mathfrak{z}\right),
$$

and this relation together with equation (4) implies

$$
Z_{\mathbf{K}}^{2}\left(\mathfrak{g}_{A}, \mathfrak{z}\right)=Z_{\mathbf{K}}^{2}\left(\mathfrak{g}_{A}, \mathfrak{z}\right)_{\text {fix }}+B_{\mathbf{K}}^{2}\left(\mathfrak{g}_{A}, \mathfrak{z}\right) .
$$

Therefore any central extension of $\mathfrak{g}_{A}$ by some $\mathfrak{z} \in \mathbf{K}$ can be described by a $\mathfrak{g}$-invariant cocycle. So let $\omega \in Z_{\mathbf{K}}^{2}\left(\mathfrak{g}_{A}, \mathfrak{z}\right)_{\text {fix }}$. Its invariance implies

$$
\begin{aligned}
\omega(1 \otimes x, a b \otimes[y, z]) & =-\omega(a \otimes y, b \otimes[z, x])-\omega(b \otimes z, a \otimes[x, y]) \\
& =-\omega(a \otimes[x, y], b \otimes z)-\omega(b \otimes z, a \otimes[x, y]) \\
& =0,
\end{aligned}
$$

and thus

$$
\omega\left(1 \otimes \mathfrak{g}, \mathfrak{g}_{A}\right)=0,
$$

since $\mathfrak{g}_{A}$ is perfect. Fixing $a, b \in A$, the map

$$
\omega_{(a, b)}: \mathfrak{g} \times \mathfrak{g} \rightarrow \mathfrak{z}:(x, y) \mapsto \omega(a \otimes x, b \otimes y)
$$

is a continuous $\mathfrak{g}$-invariant bilinear map and therefore has to be symmetric, since $\mathfrak{g}$ does not possess any non-zero skew-symmetric $\mathfrak{g}$-invariant bilinear form (cf. [12]). In view of Lemma 15, there exists a unique continuous linear map $\bar{\omega}_{(a, b)}: V(\mathfrak{g}) \rightarrow \mathfrak{z}$ satisfying $\omega_{(a, b)}=\bar{\omega}_{(a, b)} \circ \kappa$. From the uniqueness of the maps $\bar{\omega}_{(a, b)},(a, b) \in A \times A$, and the continuity of $\omega$, we deduce the existence of a continuous linear map

$$
\eta: A \otimes_{\mathbf{F}} A \rightarrow \operatorname{Lin}(V(\mathfrak{g}), \mathfrak{z})
$$

satisfying

$$
\eta(a \otimes b)(\kappa(x, y))=\omega(a \otimes x, b \otimes y) .
$$

Now the skew-symmetry of $\omega$ together with the symmetry of $\kappa$ and the fact that $\operatorname{im}(\kappa)$ generates $V(\mathfrak{g})$ as a vector space imply that $\eta$ is skew-symmetric. Using the invariance of $\kappa$, the fact that $\omega$ is a 2-cocycle yields

$$
\eta(a b \otimes c+b c \otimes a+c a \otimes b)(\kappa([x, y], z))=0
$$


for all $a, b, c \in A$ and all $x, y, z \in \mathfrak{g}$. Since $\mathfrak{g}$ is perfect and $\operatorname{im}(\kappa)$ is generating, we conclude from the latter equation that $\eta$ vanishes on all expressions of the form

$$
a b \otimes c+b c \otimes a+c a \otimes b \in A \otimes \mathbf{F} A .
$$

From (5) it follows that $\eta$ vanishes on $1 \otimes A$ and since, in view of Lemma 5 , the elements of the form

$$
a(1 \otimes b-b \otimes 1)(1 \otimes c-c \otimes 1)=a \otimes b c-a b \otimes c-a c \otimes b+a b c \otimes 1 \in A \otimes \mathbf{F} A
$$

generate a dense subset of $\overline{I^{2}}$ (recall that $I$ was defined to be the kernel of the multiplication map $\mu: A \otimes_{\mathbf{F}} A \rightarrow A$ ), we see that $\eta$ vanishes on $\overline{I^{2}}$ and hence induces a continuous linear map

$$
\bar{\eta}: \Omega_{\mathbf{K}}^{1}(A) \rightarrow \operatorname{Lin}(V(\mathfrak{g}), \mathfrak{z})
$$

For this map we have

$$
\bar{\eta}\left(d_{A}(a)\right)=\eta(1 \otimes a-a \otimes 1)=2 \eta(1 \otimes a)=0,
$$

whence it factors to a continuous linear map

$$
\xi: \Omega_{\mathbf{K}}^{1}(A) \overline{d_{A}(A)} \rightarrow \operatorname{Lin}(V(\mathfrak{g}), \mathfrak{z}) .
$$

In view of the canonical isomorphism

$$
\operatorname{Lin}_{c}\left(\Omega_{\mathbf{K}}^{1}(A) / \overline{d_{A} A}, \operatorname{Lin}(V(\mathfrak{g}), \mathfrak{z})\right) \cong \operatorname{Lin}_{c}\left(\left(\Omega_{\mathbf{K}}^{1}(A) / \overline{d_{A} A}\right) \otimes V(\mathfrak{g}), \mathfrak{z}\right),
$$

where $\operatorname{Lin}_{c}$ means continuous linear maps, we can consider $\xi$ as a continuous linear map $\mathfrak{z}_{A} \rightarrow \mathfrak{z}$, and with this identification the above calculations yield $\omega=-\xi \circ \omega_{A}$.

Having Theorem 9 in mind, we consider the special case $A:=C^{\infty}(M)$. We put $\mathfrak{g}_{M}:=C^{\infty}(M, \mathfrak{g}) \cong \mathfrak{g}_{A}$ and in order to obtain a convenient description of the universal central extension of $\mathfrak{g}_{M}$ we set $\mathfrak{z} M:=\Omega^{1}(M) / d A$, and define a continuous 2-cocycle $\omega_{M}$ on $\mathfrak{g}_{M}$ by

$$
\omega_{M}(f \otimes x, g \otimes y):=\kappa_{\mathfrak{g}}(x, y)[f d g] \in \mathfrak{z} M,
$$

where $\kappa_{\mathfrak{g}}$ denotes the Killing form of $\mathfrak{g}$. As $d A$ is the annihilator of all continuous linear functionals $\int_{\alpha}: \Omega^{1}(M) \rightarrow \mathbb{R}, \alpha \in C^{\infty}\left(\mathbb{S}^{1}, M\right)$, it is closed in $\Omega^{1}(M)$ and we have $\mathfrak{z} M \cong \mathfrak{z}_{A}$. Since, furthermore, in case $\mathfrak{g}$ is simple all invariant symmetric bilinear forms on $\mathfrak{g}$ are multiples of the Killing form, we get the following consequence of Theorem 16 which generalizes Proposition 4.2.8 in [16]:

17. Corollary. If the Lie algebra $\mathfrak{g}$ is simple, then the Lie algebra $\widetilde{\mathfrak{g}}_{M}:=\mathfrak{g}_{M} \oplus_{\omega_{M}} \mathfrak{z} M$ is a universal central extension of $\mathfrak{g}_{M}$.

Analogous to Corollary 17 we obtain another interesting consequence of Theorem 16 by taking Theorem 11 into account. We put $A_{c}:=C_{c}^{\infty}(M), \mathfrak{g}_{M, c}:=C_{c}^{\infty}(M, \mathfrak{g}) \cong \mathfrak{g}_{A}$, $\mathfrak{z}_{M, c}:=\Omega_{c}^{1}(M) / d A_{c}$ and define a continuous 2-cocycle $\omega_{M, c}$ on $\mathfrak{g}_{M, c}$ by

$$
\omega_{M, c}(f \otimes x, g \otimes y):=\kappa_{\mathfrak{g}}(x, y)[f d g] \in \mathfrak{z} M, c .
$$

For the associated central extension of $\mathfrak{g}_{M, c}$ we then have the following result:

18. CoROLlary. If the Lie algebra $\mathfrak{g}$ is simple, then the Lie algebra $\widetilde{\mathfrak{g}}_{M, c}:=\mathfrak{g}_{M, c} \oplus_{\omega_{M, c}}$ $\mathfrak{z} M, c$ is a universal central extension of $\mathfrak{g}_{M, c}$. 


\section{References}

[1] F. F. Bonsall and J. Duncan, Complete Normed Algebras, Ergeb. Math. 80, Springer, Berlin, 1973.

[2] A. Connes, Noncommutative differential geometry, IHES 62 (1985).

[3] B. L. Feigin, On the cohomology of the Lie algebra of vector fields and of the current algebra, Selecta Math. Soviet. 7 (1988), 49-62.

[4] H. Glöckner, Spaces of sections as topological modules, manuscript, Göttingen, 2001.

[5] A. Grothendieck, Produits tensoriels topologiques et espaces nucléaires, Mem. Amer. Math. Soc. 16 (1955).

[6] A. Haddi, Homologie des algèbres de Lie étendues à une algèbre commutative, Comm. Algebra 20 (1992), 1145-1166.

[7] K. H. Hofmann and S. A. Morris, The Structure of Compact Groups, de Gruyter, Berlin, 1998.

[8] H. Jarchow, Locally Convex Spaces, B. G. Teubner, Stuttgart, 1981.

[9] G. Köthe, Topological Vector Spaces I, Grundlehren Math. Wiss. 159, Springer, Berlin, 1969.

[10] P. Maier and K.-H. Neeb, Central extensions of current groups, manuscript, TU Darmstadt, 2001.

[11] R. V. Moody and A. Pianzola, Lie Algebras With Triangular Decomposition, Series of Monographs and Advanced Texts, Canad. Math. Soc., 1993.

[12] A. Medina and P. Revoy, Algèbres de Lie orthogonales, modules orthogonaux, Comm. Algebra 27 (1993), 2295-2315.

[13] K.-H. Neeb, Central extensions of infinite-dimensional Lie groups, Preprint 2084, TU Darmstadt, 2000.

[14] K.-H. Neeb, Infinite-dimensional Lie groups and their representations, Preprint 2102, TU Darmstadt, 2000.

[15] K.-H. Neeb, Universal central extensions of Lie groups, Preprint 2150, TU Darmstadt, 2001.

[16] A. Pressley and G. Segal, Loop Groups, Oxford Math. Monographs, Oxford, 1986.

[17] F. Treves, Topological Vector Spaces, Distributions and Kernels, Academic Press, 1967.

[18] P. Zusmanovich, The second homology group of current Lie algebras, Astérisque 226 (1994). 